\title{
A systematic review of neuroimaging and acute cannabis exposure in age-of-risk for psychosis
}

\author{
Lani Cupo $\mathbb{B}^{1,2}$, Eric Plitman 2,3 , Elisa Guma ${ }^{1,2}$ and M. Mallar Chakravarty 1,2,3,4
}

\begin{abstract}
Acute exposure to cannabis has been associated with an array of cognitive alterations, increased risk for neuropsychiatric illness, and other neuropsychiatric sequelae including the emergence of acute psychotic symptoms. However, the brain alterations associating cannabis use and these behavioral and clinical phenotypes remains disputed. To this end, neuroimaging can be a powerful technique to non-invasively study the impact of cannabis exposure on brain structure and function in both humans and animal models. While chronic exposure studies provide insight into how use may be related to long-term outcomes, acute exposure may reveal interesting information regarding the immediate impact of use and abuse on brain circuits. Understanding these alterations could reveal the connection with symptom dimensions in neuropsychiatric disorders and, more specifically with psychosis. The purpose of the present review is to: 1) provide an update on the findings of pharmacological neuroimaging studies examining the effects of administered cannabinoids and 2) focus the discussion on studies that examine the sensitive window for the emergence of psychosis. Current literature indicates that cannabis exposure has varied effects on the brain, with the principal compounds in cannabis (delta-9-tetrahydrocannabinol and cannabidiol) altering activity across different brain regions. Importantly, we also discovered critical gaps in the literature, particularly regarding sexdependent responses and long-term effects of chronic exposure. Certain networks often characterized as dysregulated in psychosis, like the default mode network and limbic system, were also impacted by THC exposure, identifying areas of particular interest for future work investigating the potential relationship between the two.
\end{abstract}

\section{Introduction}

In recent years there has been a surge in public policy decriminalizing or legalizing recreational cannabis use worldwide $^{1,2}$. In spite of these changing norms, our understanding of the mental health consequences of cannabis exposure remain inconclusive. From a clinical standpoint, there is an emerging consensus on how cannabis may confer some therapeutic benefits (treatments for chronic pain and glaucoma $)^{3,4}$, and may also increase risk for adverse mental health outcomes (major mental illnesses and associated symptomatology $)^{5}$. Specifically, cannabis use has been associated with increased risk for

\footnotetext{
Correspondence: Lani Cupo (lani.cupo@mail.mcgill.ca) or

M. Mallar Chakravarty (mallar@cobralab.ca)

${ }^{1}$ Integrated Program in Neuroscience, McGill University, Montreal, QC, Canada

${ }^{2}$ Computational Brain Anatomy (CoBrA) Laboratory, Cerebral Imaging Center,

Douglas Research Centre Verdun, Montreal, QC, Canada
}

Full list of author information is available at the end of the article depressive $^{6}$ and anxiety disorders ${ }^{7}$, and, central to this review, psychosis spectrum disorders ${ }^{8}$. Cannabis use initiated during early adolescence confers the greatest risk for adult psychosis ${ }^{9}$, and dose-dependent cannabis use has been associated with an increased likelihood of developing psychosis and schizophrenia ${ }^{8}$ while short-term cannabis use has been associated with increases in psychotic-like symptoms, such as altered perception and anxiety ${ }^{10}$. Risk during adolescence could in part be conferred from critical periods of development in neurotransmitters. Development of the GABA-ergic ( $\gamma$-aminobutyric acid) system during adolescence has been associated with response inhibition and working memory ${ }^{11}$. During the same time period, there occurs pruning of glutamatergic neurons, and reductions in innervation in the dopaminergic system during typical development ${ }^{11}$.

While cannabis contains many compounds responsible for various physiological effects, tetrahydrocannabinol 
(THC) is the psychoactive component most associated with psychotomimetic effects ${ }^{12}$. THC binds native cannabinoid receptors, such as G-protein coupled receptors like CB1, which acts as a receptor for endocannabinoids like anandamide ${ }^{12}$. CB1 receptors are distributed in various brain regions, and expressed on the presynaptic axon terminals of different types of neurons including GABAergic and glutamatergic neurons ${ }^{13}$. As an inhibitory neurotransmitter, active GABA-ergic synapses reduce the likelihood that postsynaptic neurons will fire. When THC or endocannabinoids bind to CB1, however, they prevent the release of GABA, permitting the postsynaptic cell to fire. An example of this prevention is dopamine, where GABA-ergic synapses control the release of dopamine into the system. Therefore, in the presence of THC, dopaminergic neurons are not prevented from firing, leading to an overabundance of dopamine. CB1 receptors are present in a high density in GABAergic axon terminals from the striatum ${ }^{14}$, potentially relating to excess dopamine in the striatum.

Increased dopamine in the striatum coincides with the dopamine hypothesis of schizophrenia as individuals with schizophrenia display excess levels of dopamine in the striatum, thought to be related to positive symptoms like hallucinations ${ }^{15}$. According to the dopamine hypothesis, patients with schizophrenia have reduced levels of dopamine in the prefrontal cortex (PFC) associated with cognitive impairments and negative symptoms like anhedonia ${ }^{15}$. The excitatory neurotransmitter, glutamate is additionally dysregulated in schizophrenia ${ }^{16,17}$, notable as glutamatergic synapses also express CB1 in the presynaptic cell. When THC binds CB1, less glutamate is released into the system, relevant to the effects seen in psychosis $^{17,18}$.

In addition to THC, other compounds in cannabis, such as cannabidiol (CBD) have a host of differential pharmacological effects on the brain with demonstrably different impacts from THC. Like the endocannabinoid 2-Arachidonoylglycerol, CBD binds CB2, a receptor that has not been as well characterized as CB1 but is largely present in the immune system ${ }^{19}$. CBD has been posited to have neuroprotective effects, reducing the effects of $\mathrm{THC}^{20}$. Previous research also suggests that exposure to cannabis with a high THC concentration increases risk compared with low-potency cannabis ${ }^{21}$. Both THC content and THC:CBD ratio in recreational cannabis seized by California law enforcement increased significantly between 1996 and $2008^{22}$.

Psychoses generally emerge earlier for men (mean age of first episode: 24.2, mean age of first negative symptom: 26.5) than for women (mean age of first episode: 27.4, mean age of first negative symptom: 41.6$)^{23}$. There is a higher incidence of schizophrenia among men (1.4:1); however, prevalence rates are similar, and women predominate at older onset ${ }^{24}$. Although the cause of the discrepancy is unknown, it has been suggested that sex hormones, such as estrogen and testosterone, may contribute to the sex differences ${ }^{24}$. Given that females are typically more sensitive to the effects of cannabis use as they relate to psychosis ${ }^{25}$, it is important to examine sex differences in cannabis response as a means to better understand this differential susceptibility

In this review, we examine studies that administer cannabinoids to better understand how mechanisms of acute exposure during adolescence and young adulthood may be implicated in changing of brain circuitry, thereby increasing risk for the emergence of psychoses. While understanding the impact of chronic use is critical, habituation makes it difficult to tease apart how cannabis alters specific brain circuits. Studies investigating chronic use are limited by confounding variables, such as concomitant tobacco ${ }^{26}$, alcohol $^{27}$, and polydrug use ${ }^{28}$, as well as shared genetic risk for psychosis and cannabis use ${ }^{29}$. By focusing on acute studies, this review reduces the confounding effects associated with repeated cannabis use. To mitigate the chance that genetic background may increase psychosis-proneness and cannabis use, we examine studies that use neuroimaging techniques to investigate how brain circuits and behavioural responses are altered following acute cannabis exposure. The alterations may reflect underlying alterations to the GABAergic, glutamatergic, and dopaminergic systems that undergo refinement during adolescence ${ }^{11}$. To capture the state of cannabis research, this review includes THC, CBD, as well as homologues of these molecules, such as tetrahydrocannabivarin ( $\mathrm{THCv})$. We synthesize the neuroimaging studies in humans and animal models that examine the effects of cannabinoid administration, both cross-sectionally and longitudinally in an age group coincident with the typical age-of-onset of psychosis (20-22; however there are additional spikes reported around 40 for women, and even some accounts of a third spike for women around 80$)^{30,31}$ to better understand the impact of cannabinoids on the brain during these sensitive periods ${ }^{23}$.

The translational neuroimaging focus of this review aims to demonstrate how whole-brain investigations of the effects of cannabis on brain function, the activity of specific receptor families, and neurochemistry can be contextualized across species. Ultimately this review seeks to reveal the state of understanding the effects of acute cannabis exposure and how this relates to the etiology of psychosis. We provide it as a reference for researchers planning projects to identify gaps in the literature and opportunities for further investigation.

\section{Methods \\ Literature search}

Neuroimaging techniques, such as functional magnetic resonance imaging (fMRI) and positron emission 
tomography (PET) are ideal for detecting the acute effects of cannabis exposure on brain function. Additionally, they permit translational approaches to research questions, including studies in both humans and non-human animals, the latter of which represents an opportunity for further research as few studies to date utilize neuroimaging techniques to study the effects of cannabinoids on non-human animal brains. We used this premise to guide our Ovid search of Medline, Embase, and PsycINFO (1980-June Week $2,2019)$ to identify articles that used neuroimaging to assay brain function in populations within an age-range relevant to the development of psychosis-like symptoms (see below) and with acute exposure to cannabinoids. Search terms included: (magnetic resonance imaging or MRI or functional magnetic resonance imaging or fMRI or positron emission tomography or PET or diffusion tensor imaging or DTI or computed tomography or CT or magnetic resonance spectroscopy or MRS) and (cannab* or tetrahydrocannabinol or THC or marijuana) and (adolescen* or develop* or teenage* or matur" or youth or young). Additionally, reference sections of major relevant reviews ${ }^{32-34}$, were reviewed for applicable articles that were potentially missed. Included studies and reviewed articles are reflected in the PRISMA flow chart (Fig. 1).

\section{Inclusion criteria}

Inclusion criteria were full-length, English-language articles that employed in vivo neuroimaging (using MRI, MRS, PET, CT, and DTI) in humans aged 14-40 (>90\% of the sample) or adolescent aged non-human animals (mouse: postnatal day [PND] $\sim 23-50^{35}$, rat: PND $\sim 28-60)^{36}$ as well as administration of synthetic or natural cannabinoids.

\section{Exclusion criteria}

Exclusion criteria for the systematic review included comorbid psychiatric disorders, administration of synthetic cannabinoid receptor agonists, or case-studies.

\section{Results}

After deduplication, the Ovid search yielded 2811 results. All titles and abstracts were reviewed by L.C., and either E.G. or E.P. (each reviewed half). Forty-four articles (40 human and four preclinical studies) met the inclusion criteria and underwent full-text assessment for eligibility (Table 1). In the following section, we provide an overview of experimental methodology and summarize behavioral results before synthesizing neuroimaging findings across studies. In order to compare networks affected by cannabis exposure and those altered across the spectrum of psychosis, studies from clinical high risk (CHR), first episode psychosis (FEP), and schizophrenia are included at the end of results sections by modality where available.

\section{Human studies}

The majority of human studies reviewed $(n=22)$ administered THC alone; ${ }^{37-58}$; methods of administration varied from vaporized $(n=11)^{39-42,46-48,53-55,57}$, to smoked $(n=4)^{50-52,59,60}$, and orally in gelatin capsules $(n=7 \text {; Table } 1)^{37,38,43,44,49,56,58}$. The second most commonly administered cannabinoid was Dronabinol, a synthetic THC often prescribed medically and reported as Marinol $(n=6)$, administered orally $[n=5]^{61-65}$ and intravenously $[n=1])^{66}$. Studies that compared THC and CBD used gelatin capsules $(n=5)^{67-71}$. Remaining studies examined the THC homologue tetrahydrocannabivarin $(n=2)^{72,73}$, Bedrobinol (a strain of cannabis with $13.5 \% \mathrm{THC}<1 \% \mathrm{CBD})(n=1)^{59}$, CBD alone $(n=1)^{74}$, or smoked cannabis without reporting CBD and THC concentrations $(n=1)^{60}$. This last study was the only one to include cannabis in its full form, while the others employed a dichotomy between THC and CBD. This work relates the human studies to relevant results from the psychosis spectrum literature $\left(\mathrm{CHR}[n=3]^{74-76}\right.$, first-episode psychosis $[n=1]^{77}$, and schizophrenia $[n=2]^{76,78}$ ).

\section{Preclinical models}

All rodent studies administered the pharmacological intervention via intraperitoneal injection. These studies examined the effect of THC $(1 \mathrm{mg} / \mathrm{kg} / \text { day for } 3 \text { weeks })^{79}$ or CB1 receptor agonists Hebrew University 210 (HU $210)^{80}$ (single injection, $\left.1 \mathrm{~mL} / \mathrm{kg}\right)^{81}$, and CP 55,940 (PND $28-38,2 \mathrm{~mL} / \mathrm{kg})^{82}$. Finally, one study examined the effects of acute and chronic HU 210 exposure on rats aged PND 35 and $70^{83}$. Both HU 210 and CP 55,940 have been demonstrated to be significantly more potent than THC, potentially limiting their comparison to cannabis use in humans ${ }^{80,84}$. One additional study in the search administered THC to Rhesus monkeys, however it falls outside of the inclusion criteria for age ${ }^{85}$.

\section{Imaging modalities}

The majority of human studies used fMRI to investigate the acute effects of cannabis exposure using resting-state fMRI (rs fMRI; $n=5)^{48,53,54,65,72}$ or event-related fMRI

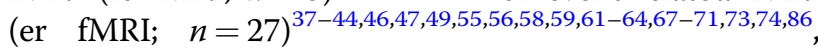
(see Table 1 for classification by task-type). Arterial spin labeling (ASL; $n=1)^{55}$ and MRS $(n=1)^{45}$ were also used. Radioligand studies included PET and single-photon emission tomography (SPET/SPECT) $(n=6)^{66}$ (see Table 1 for summary of tracers).

The three rat studies used PET to examine either glucose metabolism using $\left[{ }^{18} \mathrm{~F}\right]$-2-fluoro-deoxyglucose $\left(\left[{ }^{18} \mathrm{~F}\right]\right.$ FDG) $(n=2)^{81,82,87}$ or dopamine receptor activity with $\left[{ }^{18} \mathrm{~F}\right]$-Fallypride ${ }^{79}$. No preclinical studies used fMRI, ASL, or $1 \mathrm{H}-\mathrm{MRS}$. 


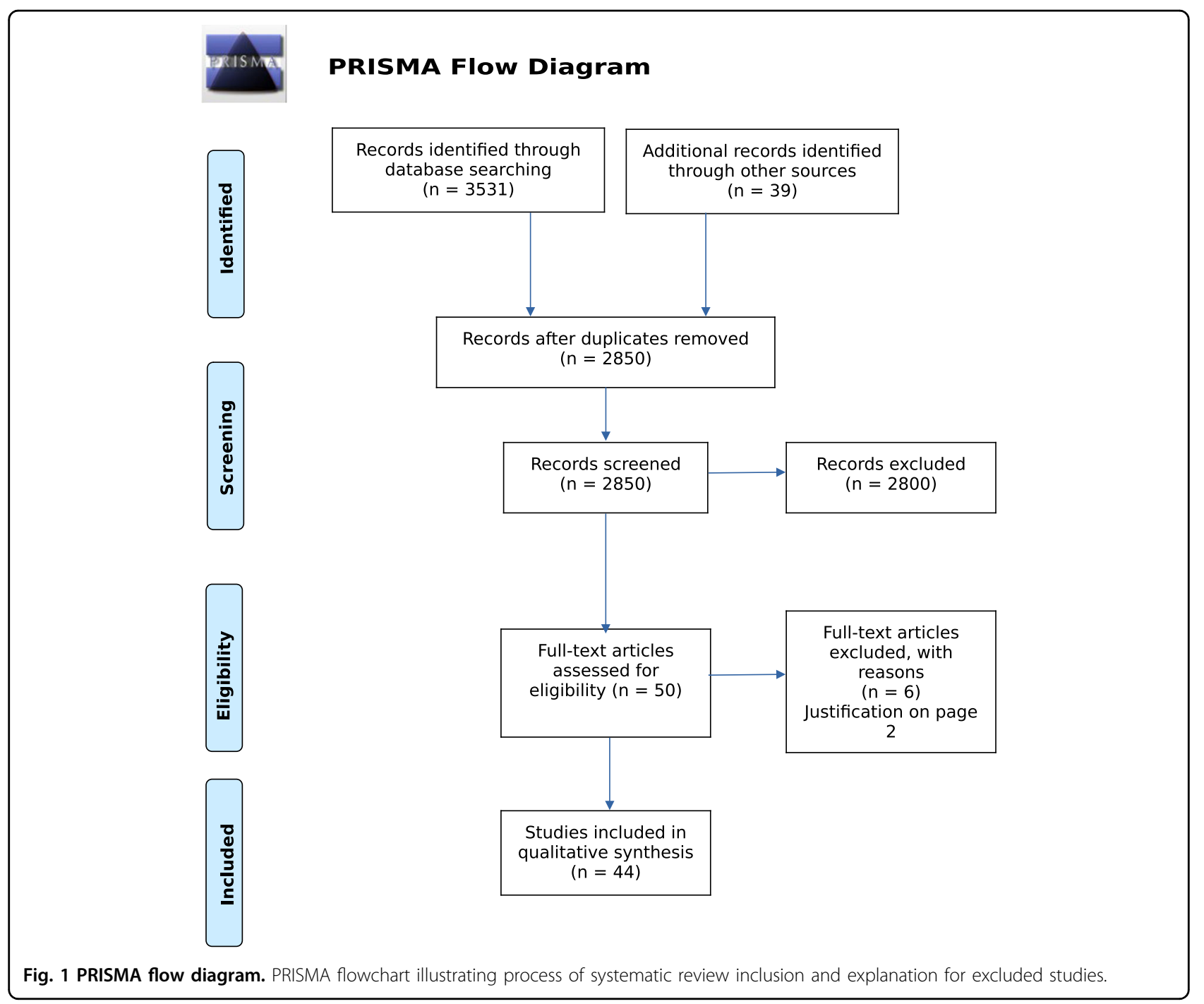

\section{Behavioral results}

Twenty-three studies reported the impact of cannabis on behavioral and psychometric assays in humans.

THC studies. The Visual Analogue Mood Scale (VAMS) was commonly used to index experiences related to "highness"/"being high", "alertness", "external perception", "internal perception", "contentedness", and "calmness" to verify the effects of THC administration $^{39-44,50,52,54,55,59,63,64,71}$. Rated with VAMS, THC exposure increased "drowsiness", "nausea", and "euphoria" 56,58 , but it reduced "alertness" $39,40,55$, "contentedness" ${ }^{\text {"0,47, "tranquility" }}$ ", and "calmness" ${ }^{41,42}$.

THC administration also increased reports of anxiety $^{37,43-45,48,50,71}$, internal and external percep$\operatorname{tion}^{40-42,47,48}$, tension and anger ${ }^{51}$, sedation ${ }^{43,45,71}$, and confusion $^{59}$. Assessments also revealed increased psychotic symptoms on the three Positive and Negative Syndrome Scale subscales (positive, negative, and general psychopathology $)^{37,43-45,69,71,88}$.
Comparison of THC and CBD administration. There was evidence for increased intoxication, anxiety, sedation, and psychotic symptoms over time in response to THC, but not to $\mathrm{CBD}^{70,86}$. Additionally, one study with a small sample (six participants) reported that three of their participants experienced acute psychotic symptoms after THC, but these symptoms were ameliorated by pretreatment with $\mathrm{CBD}^{68}$. Interpretation of the results of CBD exposure should be considered in the context of small, homogenous participant samples.

Taken together, these studies provide evidence that THC increases psychotic symptoms, anxiety, confusion, and sedation, while simultaneously reducing alertness, calmness, and contentedness. By contrast, CBD may be protective against these behavioral features.

\section{Biometric results}

Studies examining biometric effects of acute cannabis exposure observed that $\mathrm{THC}$ exposure increased heart 


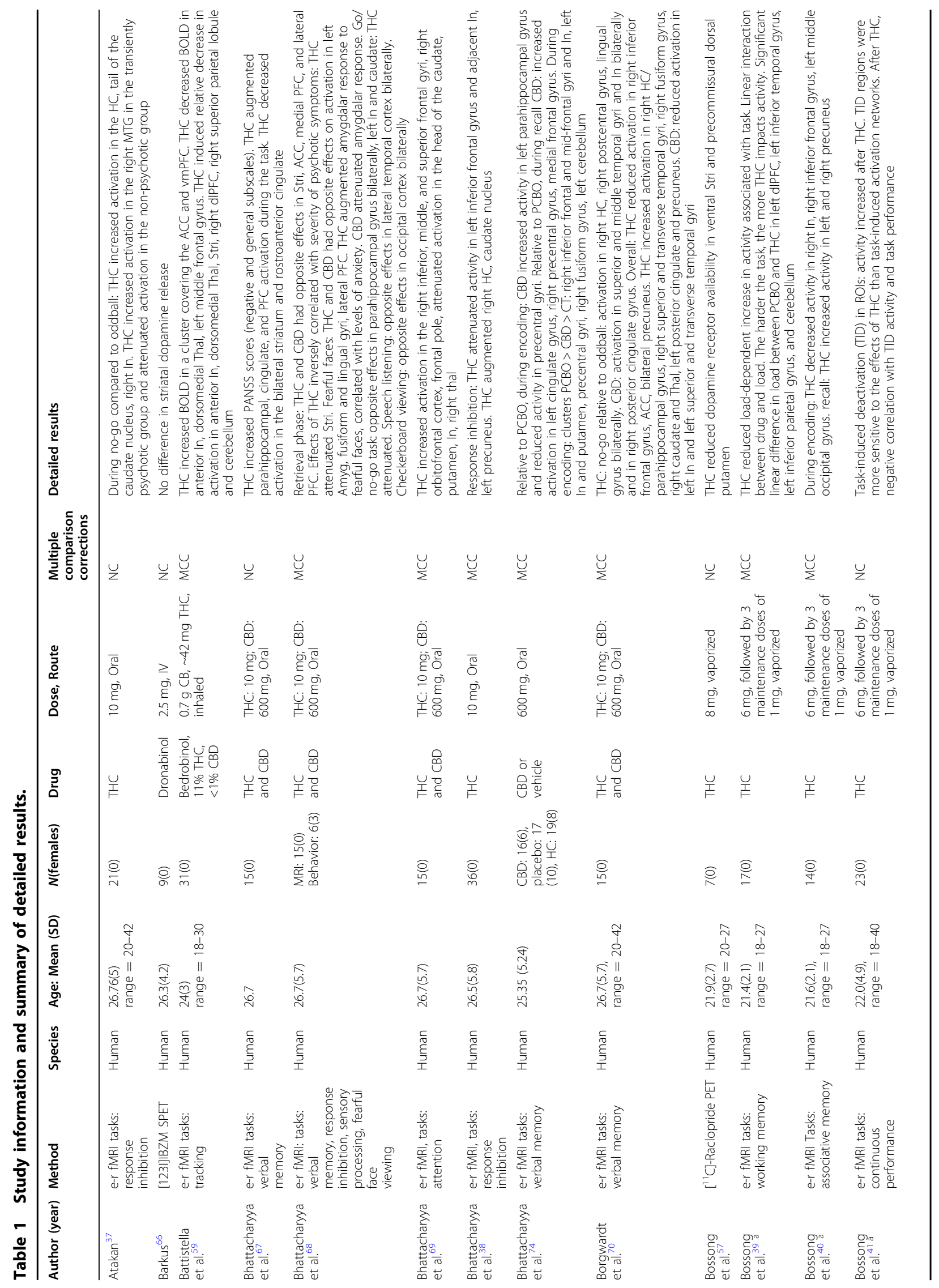




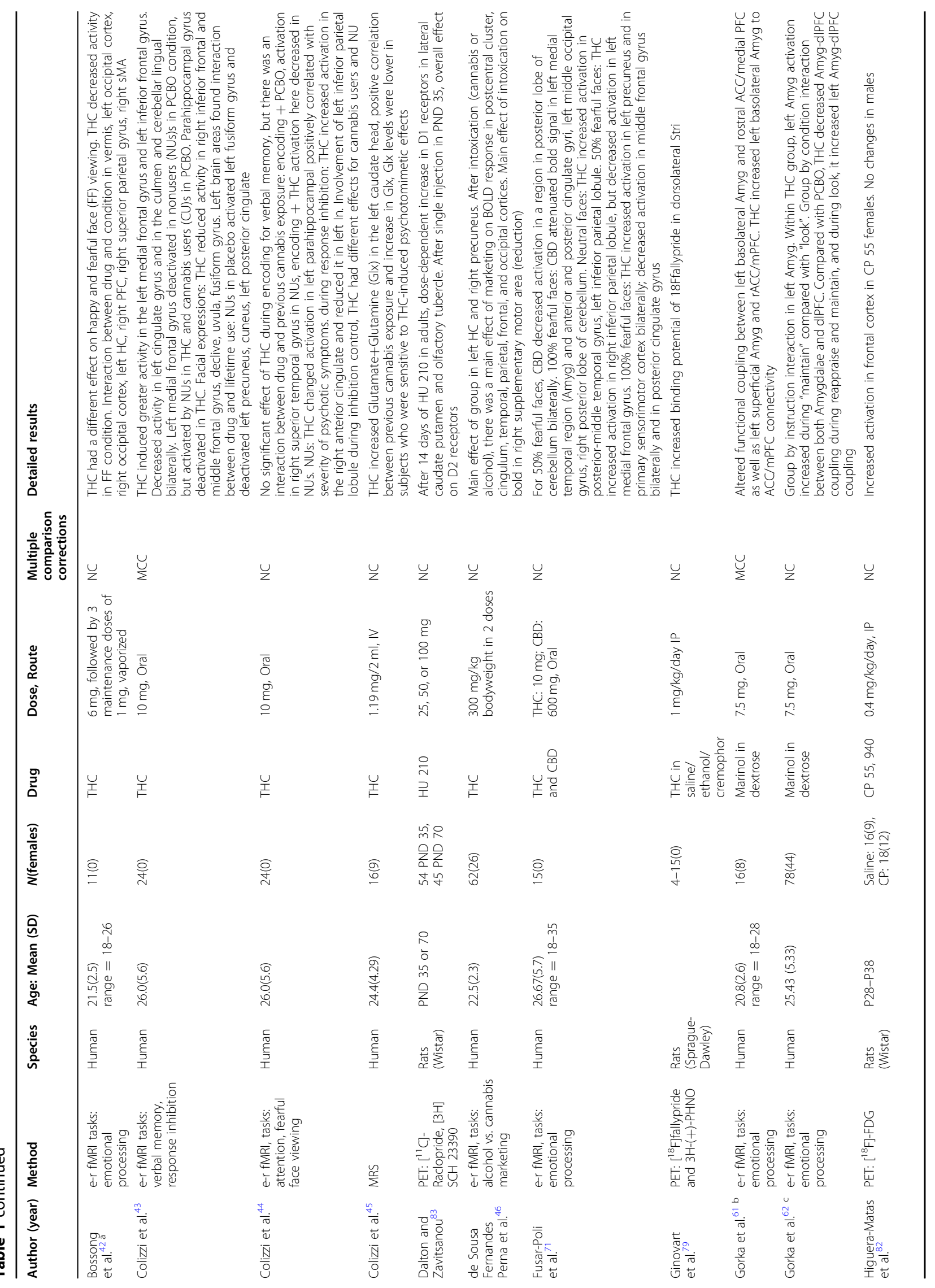




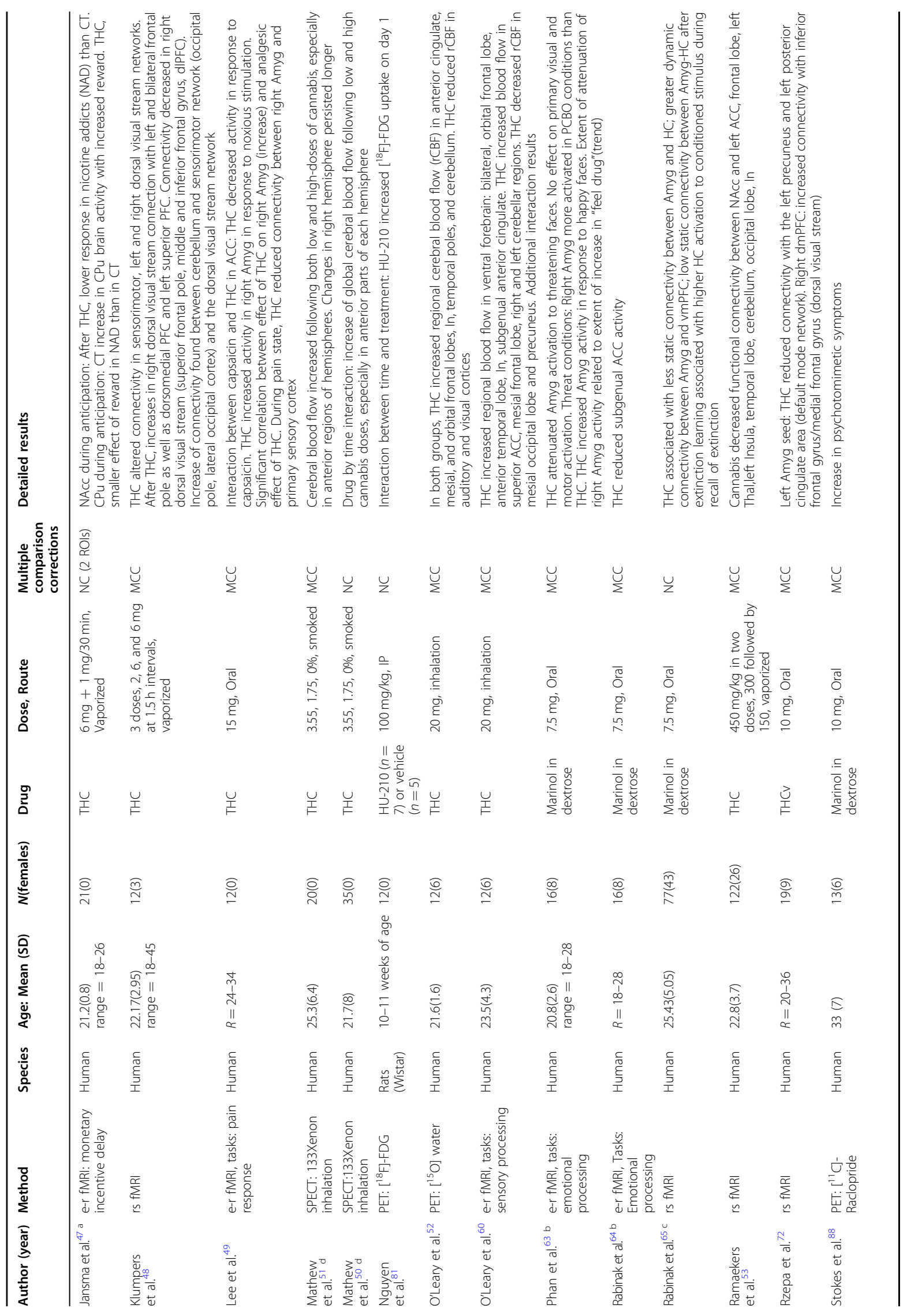




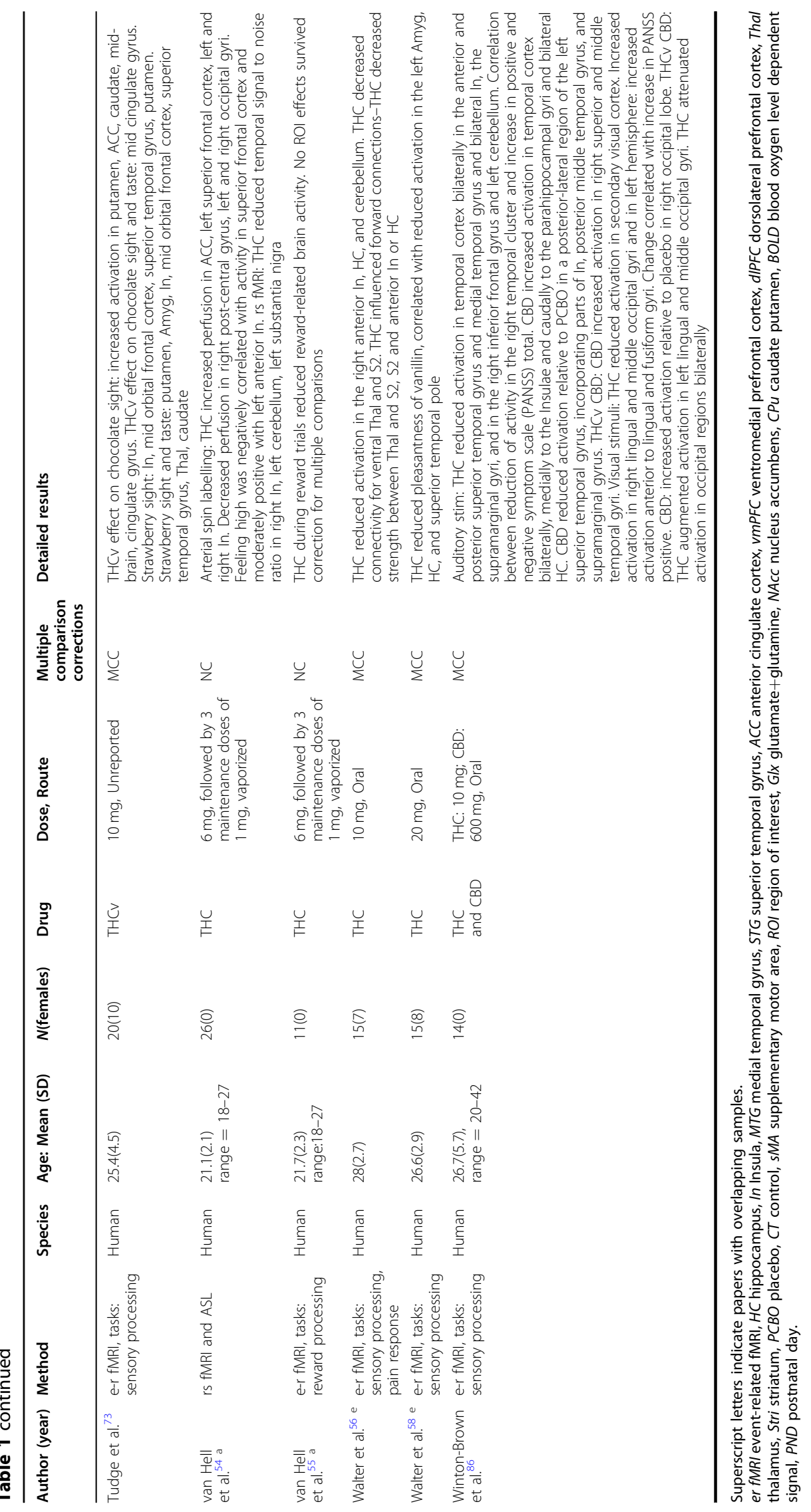


rate $^{39,40,45,48,52,54,55}$ and blood pressure ${ }^{41,42}$. Further, reports of increased cortisol levels complement selfreports of increased levels of anxiety and tension ${ }^{48}$. Meanwhile, prolactin levels were reduced, possibly related to increased dopamine activity ${ }^{48,89}$.

\section{Neuroimaging studies}

First, we report PET, rs and er fMRI, ASL, and MRS studies in humans; we further organize er fMRI studies by task type: emotional processing, memory, response inhibition, and sensory processing and examine those that do not cleanly fit into these categories. The final section investigates the preclinical studies together. Figure 2 provides a visualization of results from rs fMRI and key er fMRI studies following THC administration. Figure 3 provides a comparison with the er fMRI studies superimposed on the rs fMRI study results. Figure 4 provides a visual representation of Risk of Bias.

\section{Radioligand studies}

Three studies employed PET to examine striatal dopamine receptor availability ${ }^{57}$ and regional cerebral blood flow $^{52,60}$. Additionally, SPET was used to examine dopamine release in the striatum ${ }^{66}$. One study also combined data from two previously published studies, and since both of the prior studies were included ${ }^{57,88}$, the third was excluded.

Eight milligram of vaporized THC reduced the binding potential of $\left[{ }^{11} \mathrm{C}\right]$ raclopride in the functionally limbic part of the ventral striatum ${ }^{57}$. However, in another study $10 \mathrm{mg}$ did not alter binding of $\left[{ }^{11} \mathrm{C}\right]$ raclopride in the striatum $^{88}$.

Twenty milligrams inhaled THC increased regional cerebral blood flow (rCBF) measured with $\left[{ }^{15} \mathrm{O}\right]$ water PET in cortical regions, and the cerebellum (see Table 1) and decreased $\mathrm{rCBF}$ in auditory and visual cortices ${ }^{52}$.

One study administered a single dose of $2.5 \mathrm{mg}$ THC via intravenous injection and compared uptake of the tracer 123I-iodobenzamide in the basal ganglia. Following THC exposure, scores in the striatum ranged from a decrease by $16 \%$ to an increase by $34 \%$ and no results were significant, even though the dosages were large enough to elicit psychotic symptoms ${ }^{66}$.

Radioligand studies in psychosis. Increased striatal dopamine synthesis assessed with PET was associated with transition from prodrome to FEP in human participants $^{90}$. Additional research suggests higher baseline striatal dopamine levels in patients with schizophrenia than healthy controls ${ }^{91}$. Following amphetamine administration, there is increased dopamine release in participants with psychosis than healthy controls ${ }^{92}$. These findings are in accordance with results suggesting THC exposure may increase striatal dopamine release ${ }^{57}$.

\section{Resting-state fMRI}

Five studies assessing rs fMRI observed divergent findings. See Table 1 for specific regions.

Reward pathways. A study examined the effects of $450 \mathrm{mg} / \mathrm{kg}$ vaporized THC on impulse control in cannabis users with bilateral nucleus accumbens seeds ${ }^{53}$. Cannabis decreased resting state functional connectivity ( $\mathrm{rs}$ fc) between the accumbens and left anterior cingulate cortex (ACC), cortex, thalamus, and cerebellum.

Fronto-Limbic pathways. The impact of $10 \mathrm{mg} \mathrm{THCv}$ exposure was examined using a seed in the left amygdala $^{72}$. Decreased connectivity with important "hub" regions such as the left precuneus and left posterior cingulate (key-default mode network [DMN] regions) was observed. THCV increased connectivity between a seed in the right dorsomedial PFC and the inferior frontal/medial frontal gyrus.

One study orally-administering $7.5 \mathrm{mg}$ Marinol used specific regions of interest (ROIs: the amygdala, hippocampus $[\mathrm{HC}]$, and ventromedial PFC [vmPFC]) correlations to examine static and dynamic $\mathrm{rs} \mathrm{f}^{65}$. Their results indicated decreased static rs fc between the amygdala and $\mathrm{HC}$, but increased dynamic rs fc between the amygdala and vmPFC.

Whole brain analysis. Using networks of interest ${ }^{48}$ and a voxel-wise technique ${ }^{48,54}$, rs fc was most altered in the right dorsal visual stream network following administration of $14 \mathrm{mg}$ vaporized $\mathrm{THC}^{48}$. Increased connectivity with this region was localized in the frontal lobe. In the right hemisphere, THC decreased $\mathrm{rs} f \mathrm{fc}$ in the right hemisphere in other regions in the frontal lobe. Finally, THC increased rs fc between the cerebellum and sensorimotor network, and between the left dorsal visual stream and the occipital cortex. The second study reported the results of nine cumulative $\mathrm{mg} \mathrm{THC}$ on temporal signal-to-noise ratio (tSNR; calculated by dividing mean blood-oxygen level dependent [BOLD] signal by its standard deviation over a time period; a measure thought to reflect greater spontaneous fluctuations and brain activity) ${ }^{54}$. THC reduced tSNR, in the right insula, left cerebellum, and substantia nigra, as hypothesized by the authors ${ }^{54}$. It is critical to note that results between the whole brain studies were markedly different, potentially due in part to the analytical techniques employed.

rs fMRI in psychosis. Rs fMRI studies in participants with a FEP reveal reduced connectivity in the DMN (dorsomedial PFC and posterior cingulate cortex (PCC)/ precuneus) as well as weaker negative correlations between the lateral temporal cortex and the medial occipital lobe ${ }^{77}$. In patients with chronic schizophrenia, functional connectivity exhibits similar patterns, with decreased strengths of connectivity in the PFC, insula, and precuneus $^{93}$. 


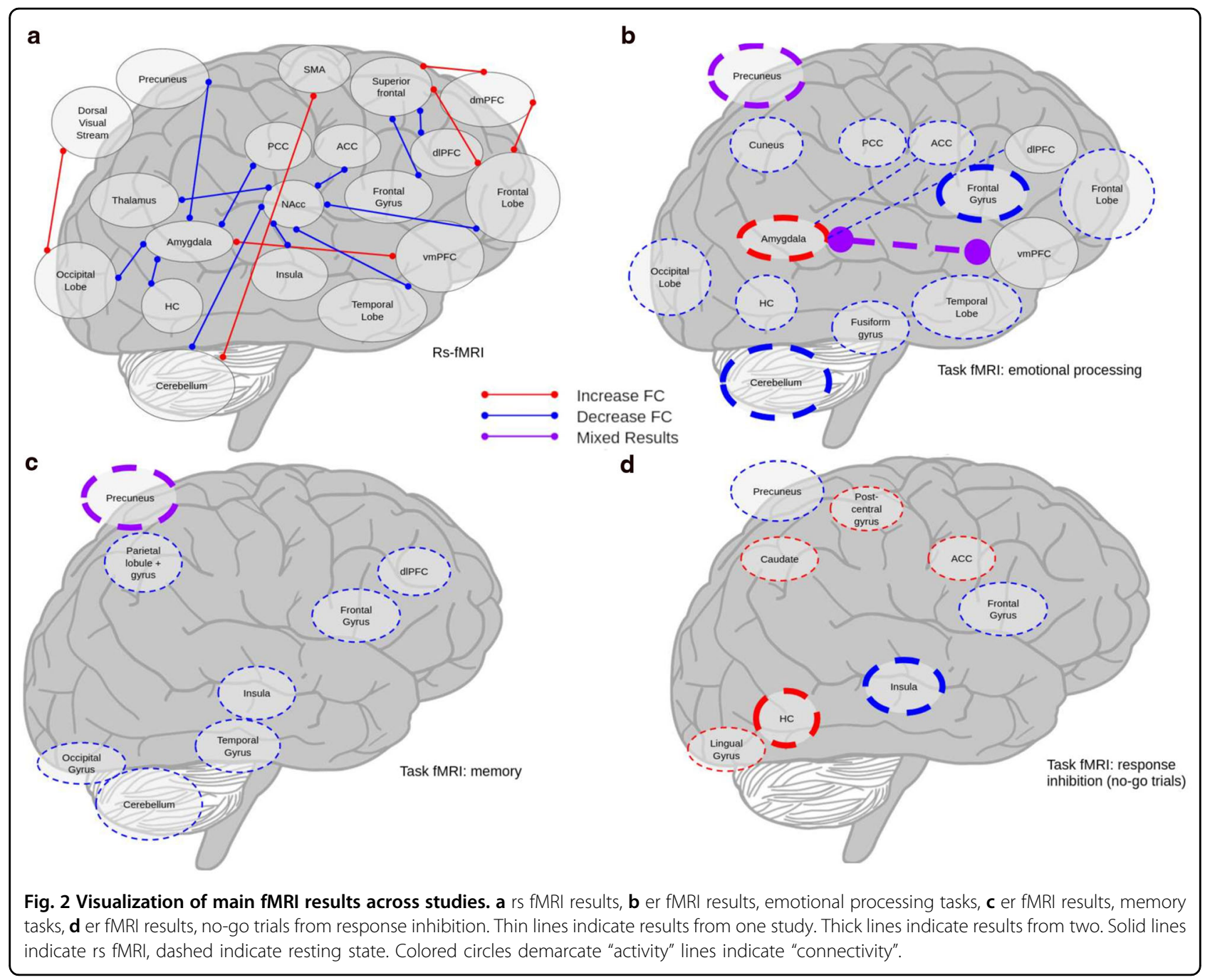

The dorsomedial PFC was implicated in both THC exposure, where increased connectivity was observed with several regions ${ }^{48,72}$, and psychosis, where decreased connectivity was observed ${ }^{77,93}$. Both THC exposure and psychosis decreased connectivity in the precuneus ${ }^{72,77,93}$, as well as the occipital lobe ${ }^{53,77}$, insula ${ }^{53,93}$. While this may indicate regions for future investigation, the variability in results may also reflect statistical noise.

\section{Event-related fMRI}

Event-related fMRI experiments used emotional processing, memory, sensory perception, and response inhibition tasks (Table 1).

Emotional processing tasks The amygdala is well-studied in the context of both THC exposure and emotional processing. A series of three studies assessed the effects of $7.5 \mathrm{mg}$ orally-administered Marinol on emotional processing in sixteen participants ${ }^{61-64}$ and found that THC attenuated amygdala activation when viewing threatening faces ${ }^{63}$.
The second study investigated rs fc between amygdala subfields and the cortex, revealing THC increased connectivity between both the amygdala and rostral ACC/ medial $\mathrm{PFC}^{62}$, but was limited to viewing threatening faces. These findings suggest that the connection between these two regions may be especially integral to social threat processing and that THC exposure increases this connection, of special interest as previous research associates perception of social threat and symptoms of paranoia ${ }^{94}$. The final study examined limbic circuitry (amygdala and ACC) engagement in response to differing valence of stimuli, and observed that THC exposure reduced activity in the subgenual ACC and did not impact amygdala activity ${ }^{64}$. These results support the view that THC decreases activity in the limbic circuit; however, the lack of effect in the amygdala provides a point of contrast to the authors' previous findings, which raises significant concerns about reproducibility and replicability.

In another task, participants were required to imagine positive contexts for negative images (e.g., reimagining a 


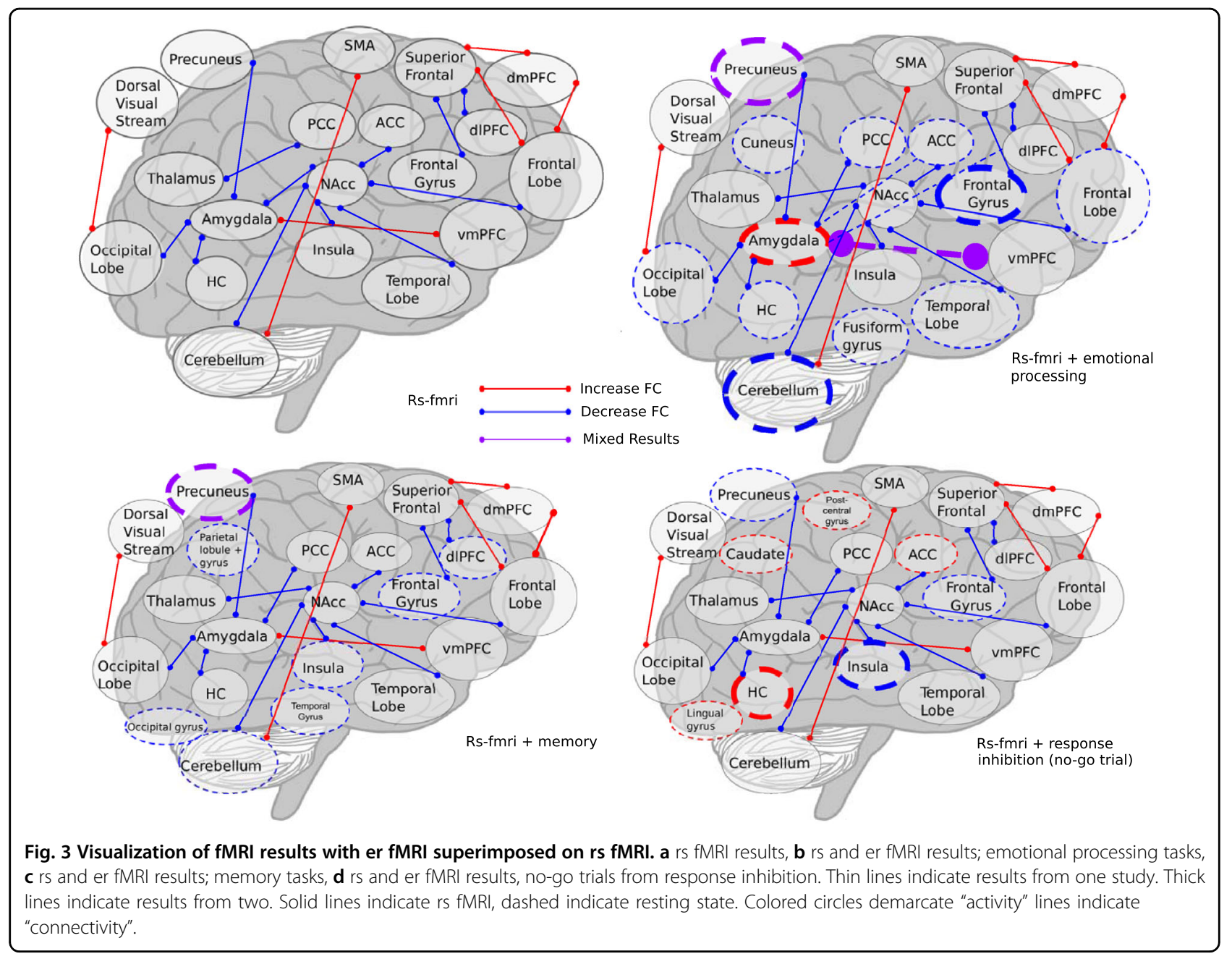

woman crying outside of a church as attending her wedding; a cognitive reappraisal task ${ }^{61}$. An increase in left amygdala activity and decrease in bilateral amygdala-dorsolateral PFC coupling was observed during the reappraisal condition following THC administration (7.5 mg) compared with placebo. When matching emotional faces, $9 \mathrm{mg}$ vaporized THC decreased activity during the fearful face condition in the cerebellum. While the decrease in activity during negative-expression-viewing is consistent with previous studies, the affected areas are inconsistent ${ }^{62-64}$.

To examine the impact of long-term cannabis use on emotional processing, one study examined fear processing in cannabis-users and nonusers ( $<5$ exposures $)^{43}$. In-study administration of $10 \mathrm{mg}$ THC reduced activity in the right inferior frontal and middle frontal gyri, medial cerebellum, and fusiform gyrus. Cannabis users had greater activity in the right cingulate gyrus and left inferior parietal lobule. These findings further support that THC reduces activity, though once again identifying some novel areas of interest (such as the cuneus), while replicating others (such as the cerebellum).
Finally, two publications from the same study population and experiment examined the differential effects of THC and CBD on emotional processing ${ }^{68,71}$. When viewing fearful faces compared with neutral faces, $600 \mathrm{mg}$ CBD reduced BOLD response in the left amygdala, left ACC, right $\mathrm{PCC}$, and right cerebellum ${ }^{71}$. Ten milligram THC exposure during fearful face viewing increased activation in the left precuneus, but decreased it in frontal and temporal regions. During fearful face viewing, THC and CBD had opposite effects, with THC and placebo increasing amygdalar activation while CBD decreased it $^{68}$. The authors also reported opposite effects in the fusiform and lingual gyri, lateral PFC, and cerebellum without specifying the directions of effects. Without more diverse samples, it is impossible to conclusively determine THC and CBD have opposite effects. Additionally, the reported results are not identical, necessitating further clarification of both methodology and the findings themselves. Visualization of the effects of THC administration during emotional processing tasks is presented in Fig. 2b. 
Green $=$ present, red $=$ absent, yellow $=$ unclear, orange $=$ not applicable

\begin{tabular}{|c|l|l|l|l|l|}
\hline Author & Double-blind? & Randomized? & Placebo-controlled? & Within-subject? & $\begin{array}{l}\text { Crossover/Counter } \\
\text {-balanced }\end{array}$ \\
\hline Atakan (2013) & & Pseudo & & & \\
\hline Barkus (2011) & & & & & \\
\hline Battistella (2013) & & & & & \\
\hline Bhattacharyya (2009) & & & & & \\
\hline Bhattacharyya (2010) & & Pseudo & & & \\
\hline Bhattacharyya (2012) & & Pseudo & & & \\
\hline Bhattacharyya (2014) & & Pseudo & & & \\
\hline Bhattacharyya (2018) & & & & & \\
\hline Borgwardt et al (2008) & & Pseudo & & & \\
\hline Bossong (2009) & & & & & \\
\hline Bossong (2012a) & & & & & \\
\hline Bossong (2012b) & & & & & \\
\hline Bossong (2013a) & & & & & \\
\hline Bossong (2013b) & & & & & \\
\hline Colizzi (2018a) & & & & & \\
\hline Colizzi (2018b) & & & & & \\
\hline Colizzi (2019) & & & & & \\
\hline Dalton (2010) & blind? & & & & \\
\hline de Sousa Fernandes & & & & & \\
\hline Fusar-Poli et al (2009) & & & & & \\
\hline Ginovart (2012) & Researchers & & & \\
\hline Gorka (2015) & & & & & \\
\hline Higuera-Matas (2008) & & & & & \\
\hline Jansmactorial & & & \\
\hline (2013) & & & & & \\
\hline
\end{tabular}

\begin{tabular}{|c|l|l|l|l|l|}
\hline Klumpers (2012) & & & & & \\
\hline Lee (2013) & & & & & \\
\hline Mathew (1992) & & & & & \\
\hline Mathew (1993) & & & & & \\
\hline Nguyen (2012) & $\begin{array}{c}\text { Researchers } \\
\text { blind? }\end{array}$ & & & & \\
\hline O'Leary (2003) & & & & & \\
\hline O'Leary (2007) & & & & & \\
\hline Phan (2008) & & & & \\
\hline Rabinak (2011) & & & & & \\
\hline Rabinak (2018) & & & & & \\
\hline Ramaekers (2016) & & & & & \\
\hline Rzepa (2015) & & & & & \\
\hline Stokes (2009) & & & & & \\
\hline Tudge (2014) & & & & & \\
\hline van Hell (2011) & & & & & \\
\hline van Hell (2012) & & & & & \\
\hline Walter (2016) & & & & & \\
\hline Walter (2017) & & & & & \\
\hline Winton-Brown (2011) & & Pseudo & & & \\
\hline
\end{tabular}

Fig. 4 Risk of bias. Assesses likelihood of bias in each paper examining for double-blind, randomized, placebo-controlled, within-subject, and crossover/counter-balanced. Green $=$ present, red $=$ absent, yellow $=$ unclear, orange $=$ not applicable.

Emotional processing in psychosis. Participants at risk for psychosis demonstrated altered activation in response to valenced faces when compared to control groups ${ }^{75}$.
Unlike controls, the high-risk group showed a relative increase in activation in response to neutral rather than sad faces in the amygdala-hippocampal complex, 
thalamus, and cuneus. The amygdala ${ }^{61-64,68,71}$ and cuneus $^{43}$ were implicated in emotional processing during cannabis exposure as well. Negatively valenced stimuli did not elicit as strong of a response in individuals with psychosis compared to neutral faces, the directionality consistent with response to $\mathrm{CBD}^{68}$, but not $\mathrm{THC}^{62,68}$.

Memory tasks Previous evidence suggests chronic cannabis use can impair memory ${ }^{95}$. Six studies investigated the impact of THC on memory $39,40,44,67,68,74$.

Verbal memory. One study demonstrated that cannabis users and nonuser controls both during $10 \mathrm{mg}$ orallyadministered THC and placebo, deactivated the right superior temporal gyrus during the task ${ }^{44}$.

Another study found that following $10 \mathrm{mg}$ THC administration, recall was associated with increased activity in the left dorsal ACC and medial PFC and decreased activity in the bilateral striatum and left rostral anterior cingulate gyrus, but found no influence of the administration of $600 \mathrm{mg}$ of $\mathrm{CBD}^{67}$. Contradictory results are published in another study reporting on the same experiment in the same participant group, where the authors reported that THC and CBD had opposite effects in the striatum, ACC, and medial and lateral PFC during retrieval, with THC decreasing activity and CBD increasing it ${ }^{68}$. The same group also studied individuals at CHR for psychosis and found that $600 \mathrm{mg}$ CBD decreased activation in the left parahippocampal gyrus during recall, but increased activation in the left cingulate gyrus, right precentral gyrus, and medial frontal gyrus ${ }^{74}$. There was a step-wise difference in activation across the three groups with the CHR group in the middle. These results provide intriguing evidence that CBD may normalize memorytask impairment for CHR populations.

Additional memory tasks. Two additional studies conducted with the same participants used the Sternberg item recognition paradigm ${ }^{40}$ and a pictorial memory task ${ }^{39}$. Difficulty of the Sternberg task can be scaled to allow for assessment of load-dependent increases in brain activity. Nine milligram of THC reduced load-dependent activity in the cortex and cerebellum ${ }^{40}$. In the pictorial memory task, THC reduced activity in the right insula, right inferior frontal gyrus, left middle occipital gyrus during encoding of images, and increased activity in the precuneus bilaterally during recall ${ }^{39}$. While the results differed in areas impacted by $\mathrm{THC}$, both studies indicate that during encoding, THC reduces activity. Differing areas of impact could be due to the respective brain-areas employed in the tasks, however without replication it is also possible that the reported results reflect properties of the methodology, rather than the drug or task. Visualization of the impact of THC on memory tasks is provided in Fig. 2c.
Memory tasks in psychosis. In a verbal memory task, during encoding, participants at risk for psychosis showed decreased activation in the frontal and parahippocampal gyri compared to healthy controls ${ }^{74}$. Surprisingly, these results align with findings that CBD decreased activation in the parahippocampal gyrus during recall in participants at CHR for psychosis ${ }^{74}$.

Response inhibition tasks Response inhibition was operationalized in a go/no-go test paradigm. In the nogo trials, $10 \mathrm{mg}$ THC administration attenuated activation in the left inferior frontal gyrus, adjacent insula, and precuneus, which were all activated following placebo administration ${ }^{38}$ conversely THC increased engagement from the right hippocampus and caudate nucleus.

One study examined the impact of previous cannabis use on response to acute exposure during response inhibition $^{44}$. Ten milligram THC increased activation in the right ACC and, similar to the above study, reduced activation in the left insula.

In a study examining the contrasting effects of $10 \mathrm{mg}$ THC and $600 \mathrm{mg}$ CBD, no-go trials following THC exposure were associated with greater activation in the right hippocampus, right postcentral gyrus, and bilateral lingual gyrus ${ }^{70}$. No-go trials in the CBD condition were associated with greater activation in the temporal gyri, insula, and PCC. While the drugs had distinct effects, they did not exhibit the same oppositional pattern present in the emotional processing studies. The findings of the go/no-go task employed in the aforementioned THC and CBD experiment were reported again in a paper highlighting the different effects of THC and $\mathrm{CBD}^{68}$. The authors reported finding opposite effects during the go/no-go in the bilateral parahippocampal gyrus, left insula, and caudate, with THC reducing activation and CBD increasing it. While the methods are reported as the same, the results differ between papers. The latter ${ }^{68}$ claims CBD and THC have opposite effects, while activation was varied in the initial paper ${ }^{70}$. Visualization of the impact of THC exposure on no-go trials is provided in Fig. 2d.

Response inhibition in psychosis. Comparing healthy controls to participants at CHR for psychosis and early schizophrenia during a go/no-go task, the right inferior frontal gyrus and bilateral dorsal ACC showed decreased activation during no-go relative to go in comparison with healthy controls, this pattern arising primarily from reduced no-go response activity ${ }^{76}$. THC also attenuated activation during no-go in the inferior frontal gyrus ${ }^{38}$, but increased activity in the $\mathrm{ACC}^{44}$.

Sensory processing Five studies examined the effects of cannabis on sensory perceptions, examining gustation ${ }^{73}$, visual and auditory stimuli ${ }^{68,86}$, and pain ${ }^{49,56}$. 
Gustation. The sole study examined how THCv impacted appetite depending on pleasant or aversive flavor and visual stimuli ${ }^{73}$. While $10 \mathrm{mg} \mathrm{THCv}$ did not change subjective stimuli ratings, it increased activity in response to the chocolate stimuli (paired visual and taste) in the caudate, midbrain, and cingulate gyrus. In response to a picture of moldy strawberries, $\mathrm{THCv}$ increased activation in the insula, frontal cortex, temporal gyrus, and putamen.

Audition. A study involving listening to neutral words read aloud demonstrated that THC reduced activity primarily in the temporal cortex, whereas CBD increased activity in the same region ${ }^{86}$. CBD also increased activity in the temporal gyri relative to THC. These results were replicated in a paper discussing the opposing effects of THC and CBD, where authors observe opposite directions of activation in the bilateral lateral temporal cortex ${ }^{68}$.

Vision. The same study investigating audition also examined the effects of cannabinoids on visual processing of checkerboard stimuli ${ }^{68,86}$. Relative to placebo, $10 \mathrm{mg}$ THC reduced activity in the secondary visual cortex, and increased activity in the lingual, occipital, and fusiform gyri whereas $600 \mathrm{mg}$ CBD increased activation in the right occipital lobe. THC increased activity in the left lingual and middle occipital gyri, also decreasing it in scattered areas of the occipital cortex and cerebellum relative to $\mathrm{CBD}$. The opposite results in the occipital lobe are also reported in the larger study comparing THC and CBD activation ${ }^{68}$.

Pain perception. Two studies examined the effect of THC on pain perception supporting the use of cannabis as an analgesic ${ }^{49,56}$. One study demonstrated that $10 \mathrm{mg}$ THC reduced activation in the right anterior insula, hippocampus, and cerebellum after inducing pain by activating trigeminal nociceptors with $\mathrm{CO}_{2}{ }^{56}$. An ROI analysis further revealed that $\mathrm{THC}$ decreased connectivity between the thalamus and secondary somatosensory cortex, which agreed with lower ratings of pain perception following THC exposure.

Fifteen microgram of THC decreased activity in the ACC in response to a topical application of capsaicin and lowered pain perception, but increased activity in the right amygdala in response to painful stimuli was correlated with the analgesic effects ${ }^{49}$. THC also reduced functional connectivity between the right amygdala and the primary sensorimotor cortex (S1) during ongoing pain, and decreased both subjective ratings of pain and limbic activity in response to painful stimuli.

Pain perception in psychosis. Patients with schizophrenia demonstrate reduced pain perception in comparison with healthy control, along with increased BOLD response in S1, but relatively reduced responsivity in the PCC, insula, and brainstem ${ }^{78}$. The analgesia reported in psychosis corresponds with that reported following cannabis exposure, as did reports of reduced activity in the insula ${ }^{56}$, however unlike individuals with psychosis, THC exposure decreased activity in $\mathrm{S}^{49}$.

Remaining tasks The remaining studies examined the effects of THC on monetary incentive delay ${ }^{47,55}$, cannabis marketing ${ }^{46}$, executive functioning ${ }^{41}$, attention ${ }^{43,69}$, and visuo-motor tracking ${ }^{59}$.

Monetary incentive delay (reward processing ${ }^{55}$ ). Nine microgram of THC reduced reward-related activity in the parietal cortex and temporal gyrus. These results indicate THC reduces responsivity to reward anticipation and presentation.

Marketing. THC $(300 \mathrm{mg} / \mathrm{kg})$ reduced BOLD signal in the right supplementary motor area in response to cannabis marketing ${ }^{46}$. Additionally, THC treatment overall reduced BOLD in the bilateral pallidum, striatum, and right caudate.

Executive functioning. Task-induced deactivation in a continuous performance task with identical pairs was observed in a network comprising the cortical regions and the cerebellum, which was more sensitive to the effects of $9 \mathrm{mg}$ THC than other networks ${ }^{41}$. These findings indicate THC may dysregulate the DMN by increasing activity during tasks.

Visual oddball detection. Two studies used the visual oddball detection task, where participants respond to presentation of visual stimuli, to assess attention ${ }^{69}$. Relative to placebo, $10 \mathrm{mg}$ THC increased activity in the right frontal gyri and frontal pole; THC also decreased activity in the right subcortical areas. CBD $(600 \mathrm{mg})$ reduced activity in the left medial PFC and increased activity in similar subcortical areas. The second study examined the impact of previous cannabis use and found that after $10 \mathrm{mg}$ THC exposure ingested orally, nonusers activated the left medial frontal gyrus, as did cannabis users after placebo ${ }^{43}$. Cannabis users in the THC condition deactivated the same area, as did nonusers in the placebo condition.

Motor control. One study examined the impact of $42 \mathrm{mg}$ inhaled THC on psychomotor control with a visuo-motor tracking test to assess the impact of THC exposure on driving ability ${ }^{59}$. THC increased BOLD response in the ACC and ventromedial PFC, however it decreased activity in the thalamus and cortical regions. Combined with results that indicate impaired tracking of the target in the task, these findings shed light on the urgent need for more research of the effects of cannabis on psychomotor activity in relation to safe driving.

\section{Arterial spin labeling}

Examining ASL, $9 \mathrm{mg}$ THC increased perfusion compared to placebo in the ACC, left superior frontal cortex and bilateral insula, and decreased perfusion in the postcentral and occipital gyri ${ }^{54}$. The increased perfusion 
associated with THC exposure may be explained by the vasodilative effects of cannabis.

\section{Magnetic resonance spectroscopy}

Ten milligram of orally-ingested THC increased rates of Glx (a pseudo-concentration of glutamate and glutamine) in the left caudate head, with the highest rates of increase in those who had the lowest levels of Glx in the placebo condition.

MRS in psychosis. Increased levels of glutamate in the dorsal caudate predicted transition to psychosis in CHR groups, and compared to healthy controls and those who did not transition, the transition group displayed higher rates of glutamate ${ }^{96}$. These findings correspond to increased rates of Glx following THC exposure ${ }^{45}$.

\section{Animal models}

Only four animal studies (all using PET) met the inclusion criteria. Radioactive tracers and rat background strains are listed in Table 1.

Nguyen et al. performed $\left[{ }^{18} \mathrm{~F}\right]$-FDG PET $15 \mathrm{~min}$ and $24 \mathrm{~h}$ following injection of $100 \mathrm{mg} / \mathrm{kg}$ HU 210 (a THC homologue) in 10-11-week old rats. They observed that HU 210 increased global uptake of $\left[{ }^{18} \mathrm{~F}\right]$-FDG only at the first timepoint, suggesting whole-brain hypermetabolism was acute and not persistent ${ }^{81}$.

Ginovart et al. administered daily $1 \mathrm{mg} / \mathrm{kg}$ THC injections for three weeks to male rats. While age was not reported, the reported weights of rats suggest that they were between 8 and 9 weeks old ${ }^{97}$. Results of the in vivo PET imaging revealed that THC increased D2 and D3 receptor availability in the dorsal striatum based on $\left[{ }^{18} \mathrm{~F}\right]$ fallypride binding. Ex vivo autoradiography confirmed these findings, but also demonstrated increases in binding in the subcortical regions ${ }^{79}$.

Finally, after a single injection in PND 35, there was an overall effect of HU 210 on D2 receptors, however there was no interaction in individual regions ${ }^{83}$.

\section{Discussion}

\section{Summary and implications}

A systematic review of the literature investigating cannabis administration and neuroimaging reveals the heterogeneity in both methodology and findings. Overall, in rs fMRI, certain findings converge, despite differing analytical approaches. After the administration of both THC and $\mathrm{THCv}$, there is increased connectivity between the dorsomedial PFC and the dorsal visual stream network both in the seed-based and whole-brain approach ${ }^{48,72}$. In order to facilitate interpretation and comparison with previous studies, future rs fMRI work should utilize multiple techniques for analysis, such as whole-brain voxel-wise analyses, seed-based approaches, and predefined ROIs, to examine in a single population which findings consistently appear across methodologies.

Event-related fMRI studies show disappointingly divergent results, for example THC both increases and decreases BOLD response in the amygdala during negatively valenced emotional stimuli ${ }^{61,63}$. Experimental design may change the effects of THC on pain sensitivity, with THC generally decreasing activity, but in different regions ${ }^{49,56}$. Small sample sizes and the absence of replication among studies limit the generalizability of results. The limited agreement among studies is illustrated in Figs. 2 and 3. In part, the lack of agreement could be due to focused analyses, such as the emphasis on the nucleus accumbens, which one study identified as a seed region ${ }^{53}$, whereas this area is not significant in studies performing whole-brain analyses. Figure 3 further demonstrates the lack of coherence among studies, examining the concurrence between rs and er fMRI studies. The diversity of results renders it difficult to draw meaningful conclusions across studies, but ultimately highlights the need for more rigorous research into the effects of cannabinoids. Given the well publicized issues with underpowered task and rs fMRI studies ${ }^{98,99}$, investigating the acute impact of cannabis exposure will require that studies be designed to be generalizable (large samples of diverse individuals, multiple-sites, and harmonized whole-brain analyses), supporting robust conclusions.

Preclinical studies represent a major opportunity for future studies as cannabis or THC can be administered experimentally either one or many times to study either short-term or chronic effects. Neuroimaging and behavior can be assessed at multiple time points and supplemented with post-mortem assays to develop a deeper characterization of the effects of cannabis exposure. As no rodent studies utilized fMRI, ASL, or $1 \mathrm{H}-\mathrm{MRS}$, they represent areas of special interest, even acknowledging challenges such as the confounding effects of anaesthesia regimens ${ }^{100}$ and obtaining high signal-to-noise ratio ${ }^{101}$. Additionally, while the preclinical studies administered cannabinoids through injections, most human studies administered it orally. Intravenous $\mathrm{THC}$ exposure mimics exposure by smoking, however following oral consumption, THC is first metabolized by the liver, reducing bioavailability ${ }^{102}$. Differences in method of exposure could limit the comparability between human and preclinical studies. This too presents a limitation to synthesis between human results, as there is heterogeneity in methods of exposure.

\section{fMRI limitations}

The majority of studies included in this review examined either rs or er fMRI, however limitations, both inherent to this methodology and in terms of study design, impose limitations on the synthesis of results, such as the small sample sizes. Only four fMRI studies include more than sixty participants. Small sample sizes run the 
risk of being under-powered, leading to greater numbers of false negatives and overestimated effect sizes ${ }^{103}$. Future research should include power analyses and adequate sample sizes to further verify early findings in the field.

\section{THC and psychosis}

A major focus of this review is the potential relationship between THC exposure and psychotic symptoms/schizophrenia. Not only does chronic cannabis use increase the risk of developing psychosis ${ }^{104}$, but also reviewed studies demonstrate acute cannabis exposure increases temporary psychotomimetic symptoms ${ }^{37,43-45,69,71}$. There is also convergence between fMRI studies in FEP and the effects of acute THC exposure, such as decreased activity in the dorsolateral $\mathrm{PFC}^{48,105}$. Additionally, most alterations were focused in the PFC and limbic areas, similar to seven other studies in this review ${ }^{39,48,53,59,61,62,68,105}$. Similar patterns of disrupted activity are seen between both pharmacological intervention with THC and in populations with FEP complementing symptomatic similarities, such as PANSS scores. Given areas of correspondence between THC administration and psychosis, future studies seeking a mechanistic connection between THC exposure and the emergence of psychosis should consider investigating the DMN (including the medial PFC, PCC, and inferior parietal lobules) ${ }^{40,41,43,55,62,65,67,69,72,106,107}$. The limbic system, comprising the cingulate cortex, parahippocampal region, hippocampus, and amygdala, was also highly impacted by $\mathrm{THC}$ and psychosis meriting further investigation $^{42,43,49,53,60-63,65,67-69,72-74,77,107}$.

\section{Sex}

Only 17 of the 39 reviewed human studies included female participants ${ }^{45,46,48,52,53,56,58,60-65,68,72,74,82}$; similarly only one of four non-human animal experiments included female rodents ${ }^{82}$. One of the groups that used the same sample for seven studies included in this review ${ }^{39-}$ $42,47,54,55$ attributed their choice of recruiting only males to the "expected interactions between hormonal cycle and brain activity patterns in women, which will flaw the design. In addition, there is evidence for sex differences in the effects of THC", citing a review of behavioral studies demonstrating sex-differences in adult rodents ${ }^{108}$. We hope that future researchers no longer cite the mysteries of having to deal with "female hormones" as an excuse for incomplete study design. Given the number of studies that adopt this philosophy, there is an urgent need for pharmacological studies involving females ${ }^{109,110}$. There is substantial evidence suggesting sex differences in prevalence and efficiency of CB1 receptors, metabolism of cannabis, and behavioral responses ${ }^{111,112}$. To incorporate this knowledge and protect participants, future studies investigating sex-differences should administer a proportional dose based on weight to avoid attrition, as five of the studies $\operatorname{did}^{46,53,79,81,82,87}$. Evidence regarding sexeffects are mixed, with some results indicating longterm behavioral changes may be greater for males than females, illustrating the need for more in-depth studies adequately powered to examine sex-differences ${ }^{113}$.

\section{Overlapping studies}

Several of the reviewed studies reported results from different tasks acquired from the same experiment, which is important to acknowledge as discussing them independently inflates sample of participants in the literature. Studies that reported on the same data set are indicated in Table 1 with matching asterisks. Additionally, ten of the studies did not indicate that they drew from overlapping samples; however, the demographic summary statistics of participants indicate that they likely are ${ }^{38,43,44,67-71,74,86}$. It is vital to weigh interpretations of these findings with knowledge that there may be limitations to generalizability and bias due to the subjects recruited possibly leading to inflated estimates of statistical significance ${ }^{114}$. Among the significant results from these studies are the opposition of THC and CBD, limiting the generalizability of the results. Judging purely by the number of papers published, the casual reader may obtain an inflated perspective on the number of neuroimaging cannabis studies. While they provide a strong foundation, the limited number of unique participants ( 733), and the homogeneity of the samples greatly compromises the generalizability of results.

\section{Conclusion}

While the effects of cannabis exposure have become a focal point for research in recent years, much remains unknown despite the rapid legalization of cannabis around the world. This paper fills an important gap by providing a systematic review of studies that administer THC, not only suggesting potential effects of acute THC exposure but also drawing attention to certain limitations confronted by the field as a whole. Future work should consider researching long-term cannabis exposure in rodents, characterizations of dose-response relationships, sex-differences in sensitivity, and differences across mechanisms of exposure, such as oral consumption versus inhalation. A deeper understanding of the potential harms and benefits of cannabis exposure in humans requires a multifaceted examination of the effects on neurodevelopment.

\section{Acknowledgements}

The authors would like to thank Dr. Gabriel A. Devenyi for his critical review of the manuscript. E.G. and M.M.C. receive salary support from Fonds de recherche du Québec - Santé and L.C. and E.P. from Healthy Brains for Healthy Lives Fellowship from McGill University. M.M.C. receives research support from the Canadian Institutes of Health Research, National Sciences and Engineering Research Council of Canada, and Healthy Brains for Healthy Lives. 


\section{Author details}

${ }^{1}$ Integrated Program in Neuroscience, McGill University, Montreal, QC, Canada. ${ }^{2}$ Computational Brain Anatomy (CoBrA) Laboratory, Cerebral Imaging Center, Douglas Research Centre Verdun, Montreal, QC, Canada. ${ }^{3}$ Department of Psychiatry, McGill University, Montreal, QC, Canada. ${ }^{4}$ Department of Biological and Biomedical Engineering, McGill University, Montreal, QC, Canada

\section{Conflict of interest}

The authors declare no competing interests.

Publisher's note

Springer Nature remains neutral with regard to jurisdictional claims in published maps and institutional affiliations.

\section{Appendix}

Bossong, Matthijs G., et al. Further human evidence for striatal dopamine release induced by administration of $\Delta$ 9-tetrahydrocannabinol (THC): selectivity to limbic striatum. Psychopharmacology 232.15 (2015): 2723-2729. Excluded because evidence synthesized two included papers.

Freeman, T. P., Pope, R. A., Wall, M. B., Bisby, J. A., Luijten, M., Hindocha, C., ... \& Morgan, C. J. (2018). Cannabis dampens the effects of music in brain regions sensitive to reward and emotion. International Journal of Neuropsychopharmacology, 21(1), 21-32. Excluded because sample fell outside of the age-range included.

Bedi, Gillinder, Martin A. Lindquist, and Margaret Haney. An fMRI-based neural signature of decisions to smoke cannabis. Neuropsychopharmacology 40.12 (2015): 2657-2665. Excluded because sample fell outside of the age-range included.

O'Leary, D. S., et al. Acute marijuana effects on rCBF and cognition: a PET study. Neuroreport 11.17 (2000): 3835-3840. Excluded because sample fell outside of the age-range included.

van Hell, Hendrika $H$., et al. Methods of the pharmacological imaging of the cannabinoid system (PhICS) study: towards understanding the role of the brain endocannabinoid system in human cognition. International Journal of Methods in Psychiatric Research 20.1 (2011): 10-27. Excluded because description of methodology without report of results (included in other papers).

Received: 3 September 2020 Revised: 6 January 2021 Accepted: 5 February 2021

Published online: 13 April 2021

\section{References}

1. Lancione, S. et al. Non-medical cannabis in North America: an overview of regulatory approaches. Public Health 178, 7-14 (2020).

2. Miech, R. A. et al. Trends in use of marijuana and attitudes toward marijuana among youth before and after decriminalization: the case of California 20072013. Int. J. Drug Policy 26, 336-344 (2015).
3. Webb, C. W. \& Webb, S. M. Therapeutic benefits of cannabis: a patient survey Hawaii J. Med. Public Health 73, 109-111 (2014).

4. MacMillan, K., MacMillan, K. M., Keddy, A. \& Furlong, J. Cannabis and glaucoma: a literature review. Dalhous. Med. J. https://doi.org/10.15273/dmj. vol46no1.9830. (2019).

5. Volkow, N. D. et al. Effects of Cannabis use on human behavior, including cognition, motivation, and psychosis: a review. JAMA Psychiatry 73, 292-297 (2016).

6. Horwood, L. J. et al. Cannabis and depression: an integrative data analysis of four Australasian cohorts. Drug Alcohol Depend. 126, 369-378 (2012).

7. Hayatbakhsh, M. R. et al. Cannabis and anxiety and depression in young adults: a large prospective study. J. Am. Acad. Child Adolesc. Psychiatry 46, 408-417 (2007).

8. Davis, G. P., Compton, M. T., Wang, S., Levin, F. R. \& Blanco, C. Association between cannabis use, psychosis, and schizotypal personality disorder: findings from the National Epidemiologic Survey on alcohol and related conditions. Schizophr. Res. 151, 197-202 (2013).

9. Arseneault, L. et al. Cannabis use in adolescence and risk for adult psychosis: longitudinal prospective study. BMJ 325, 1212-1213 (2002).

10. D'Souza, D. C. et al. The psychotomimetic effects of intravenous delta-9tetrahydrocannabinol in healthy individuals: implications for psychosis. Neuropsychopharmacology 29, 1558-1572 (2004).

11. Kilb, W. Development of the GABAergic system from birth to adolescence. Neuroscientist 18, 613-630 (2012)

12. Bloomfield, M. A. P., Ashok, A. H., Volkow, N. D. \& Howes, O. D. The effects of $\triangle 9$-tetrahydrocannabinol on the dopamine system. Nature 539, 369-377 (2016)

13. Chiu, C. Q., Puente, N., Grandes, P. \& Castillo, P. E. Dopaminergic modulation of endocannabinoid-mediated plasticity at GABAergic synapses in the prefrontal cortex. J. Neurosci. 30, 7236-7248 (2010).

14. Herkenham, M. et al. Characterization and localization of cannabinoid receptors in rat brain: a quantitative in vitro autoradiographic study. J. Neu rosci. 11, 563-583 (1991).

15. Toda, M. \& Abi-Dargham, A. Dopamine hypothesis of schizophrenia: making sense of it all. Curr. Psychiatry Rep. 9, 329-336 (2007).

16. Howes, O., McCutcheon, R. \& Stone, J. Glutamate and dopamine in schizophrenia: an update for the 21st century. J. Psychopharmacol. 29, 97-115 (2015).

17. Plitman, E. et al. Glutamate-mediated excitotoxicity in schizophrenia: a review. Eur. Neuropsychopharmacol. 24, 1591-1605 (2014).

18. Nudmamud-Thanoi, S. et al. in Neuropsychiatric Disorders: An Integrative Approach 281-285 (Springer, 2007).

19. Silvestri, C. \& Di Marzo, V. The endocannabinoid system in energy homeostasis and the etiopathology of metabolic disorders. Cell Metab. 17, 475-490 (2013).

20. Campos, A. C., Fogaça, M. V., Sonego, A. B. \& Guimarães, F. S. Cannabidiol, neuroprotection and neuropsychiatric disorders. Pharm. Res. 112, 119-127 (2016).

21. Di Forti, M. et al. The contribution of cannabis use to variation in the incidence of psychotic disorder across Europe (EU-GEI): a multicentre casecontrol study. Lancet Psychiatry 6, 427-436 (2019).

22. Burgdorf, J. R., Kilmer, B. \& Pacula, R. L. Heterogeneity in the composition of marijuana seized in California. Drug Alcohol Depend. 117, 59-61 (2011).

23. Veen, N. D. et al. Cannabis use and age at onset of schizophrenia. Am. J. Psychiatry 161, 501-506 (2004).

24. Abel, K. M., Drake, R. \& Goldstein, J. M. Sex differences in schizophrenia. Int. Rev. Psychiatry 22, 417-428 (2010)

25. Crane, N. A., Schuster, R. M., Fusar-Poli, P. \& Gonzalez, R. Effects of cannabis on neurocognitive functioning: recent advances, neurodevelopmental influences, and sex differences. Neuropsychol. Rev. 23 117-137 (2013)

26. Bélanger, R. E., Akre, C., Kuntsche, E., Gmel, G. \& Suris, J.-C. Adding tobacco to cannabis its frequency and likely implications. Nicotine Tob. Res. 13, 746-750 (2011)

27. Pape, H., Rossow, I. \& Storvoll, E. E. Under double influence: assessment of simultaneous alcohol and cannabis use in general youth populations. Drug Alcohol Depend. 101, 69-73 (2009).

28. Van Dam, N. T., Earleywine, M. \& DiGiacomo, G. Polydrug use, cannabis, and psychosis-like symptoms. Hum. Psychopharmacol. 23, 475-485 (2008).

29. Power, R. A. et al. Genetic predisposition to schizophrenia associated with increased use of cannabis. Mol. Psychiatry 19, 1201-1204 (2014). 
30. Hare, E. et al. Heritability of age of onset of psychosis in schizophrenia. Am. J. Med. Genet. B 153B, 298-302 (2010).

31. Köhler, S. et al. Evidence that better outcome of psychosis in women is reversed with increasing age of onset: a population-based 5-year follow-up study. Schizophr. Res. 113, 226-232 (2009).

32. Batalla, A. et al. Neuroimaging studies of acute effects of THC and CBD in humans and animals: a systematic review. Curr. Pharm. Des. 20, 2168-2185 (2014).

33. Crocker, C. E., Cookey, J. \& Tibbo P. G. in Handbook of Cannabis and Related Pathologies (ed. Preedy, V. R.) 345-356 (Academic Press, 2017).

34. Meruelo, A. D., Castro, N., Cota, C. I. \& Tapert, S. F. Cannabis and alcohol use, and the developing brain. Behav. Brain Res. 325, 44-50 (2017).

35. Brust, V., Schindler, P. M. \& Lewejohann, L. Lifetime development of behavioural phenotype in the house mouse (Mus musculus). Front. Zool. 12, S17 (2015).

36. Spear, L. P. The adolescent brain and age-related behavioral manifestations. Neurosci. Biobehav. Rev. 24, 417-463 (2000).

37. Atakan, Z. et al. Cannabis affects people differently: inter-subject variation in the psychotogenic effects of $\Delta 9$-tetrahydrocannabinol: a functional magnetic resonance imaging study with healthy volunteers. Psychol. Med. 43, 1255-1267 (2013).

38. Bhattacharyya, S. et al. Protein kinase B (AKT1) genotype mediates sensitivity to cannabis-induced impairments in psychomotor control. Psychol. Med. 44, 3315-3328 (2014).

39. Bossong, M. G. et al. Effects of $\Delta$-tetrahydrocannabinol administration on human encoding and recall memory function: a pharmacological FMRI study. J. Cogn. Neurosci. 24, 588-599 (2012).

40. Bossong, M. G. et al. Effects of $\delta 9$-tetrahydrocannabinol on human working memory function. Biol. Psychiatry 71, 693-699 (2012).

41. Bossong, M. G. et al. Default mode network in the effects of $\Delta 9$-Tetrahydrocannabinol (THC) on human executive function. PLOS ONE 8, e70074 (2013).

42. Bossong, M. G. et al. The endocannabinoid system and emotional processing: a pharmacological fMRI study with $\triangle 9$-tetrahydrocannabinol. Eur. Neuropsychopharmacol. 23, 1687-1697 (2013).

43. Colizzi, M. et al. Previous cannabis exposure modulates the acute effects of delta-9-tetrahydrocannabinol on attentional salience and fear processing. Exp. Clin. Psychopharmacol. 26, 582-598 (2018).

44. Colizzi, M. et al. Modulation of acute effects of delta-9-tetrahydrocannabinol on psychotomimetic effects, cognition and brain function by previous cannabis exposure. Eur. Neuropsychopharmacol. 28, 850-862 (2018).

45. Colizzi, M. et al. Delta-9-tetrahydrocannabinol increases striatal glutamate levels in healthy individuals: implications for psychosis. Mol. Psychiatry https:/ doi.org/10.1038/s41380-019-0374-8. (2019).

46. de Sousa Fernandes Perna, E. B. et al. Brain reactivity to alcohol and cannabis marketing during sobriety and intoxication. Addict. Biol. 22, 823-832 (2017).

47. Jansma, J. M. et al. THC reduces the anticipatory nucleus accumbens response to reward in subjects with a nicotine addiction. Transl. Psychiatry $\mathbf{3}$ e234 (2013)

48. Klumpers, L. E. et al. Manipulating brain connectivity with 89 -tetrahydrocannabinol: a pharmacological resting state FMRI study. Neuroimage 63, 1701-1711 (2012).

49. Lee, M. C. et al. Amygdala activity contributes to the dissociative effect of Cannabis on pain perception. Pain 154, 124-134 (2013).

50. Mathew, R. J. \& Wilson, W. H. Acute changes in cerebral blood flow after smoking marijuana. Life Sci. 52, 757-767 (1993).

51. Mathew, R. J., Wilson, W. H., Humphreys, D. F., Lowe, J. V. \& Wiethe, K. E. Regional cerebral blood flow after marijuana smoking. J. Cereb. Blood Flow. Metab. 12, 750-758 (1992).

52. O'Leary, D. S. et al. Marijuana alters the human cerebellar clock. Neuroreport 14, 1145-1151 (2003).

53. Ramaekers, J. G. et al. Cannabis and cocaine decrease cognitive impulse control and functional corticostriatal connectivity in drug users with low activity DBH genotypes. Brain Imaging Behav. 10, 1254-1263 (2016).

54. van Hell, H. H. et al. Evidence for involvement of the insula in the psychotropic effects of THC in humans: a double-blind, randomized pharmacological MRI study. Int. J. Neuropsychopharmacol. 14, 1377-1388 (2011).

55. van Hell, H. H. et al. Involvement of the endocannabinoid system in reward processing in the human brain. Psychopharmacology 219, 981-990 (2012).
56. Walter, C. et al. Brain mapping-based model of $\Delta(9)$-tetrahydrocannabinol effects on connectivity in the pain matrix. Neuropsychopharmacology 41, 1659-1669 (2016).

57. Bossong, M. G. et al. Delta 9-tetrahydrocannabinol induces dopamine release in the human striatum. Neuropsychopharmacology 34, 759-766 (2009).

58. Walter, C. et al. Effects of oral $\Delta 9$-tetrahydrocannabinol on the cerebral processing of olfactory input in healthy non-addicted subjects. Eur. J. Clin. Pharm. 73, 1579-1587 (2017).

59. Battistella, G. et al. Weed or wheel! FMRI, behavioural, and toxicological investigations of how cannabis smoking affects skills necessary for driving. PLOS ONE 8, e52545 (2013).

60. O'leary, D. S. et al. Effects of smoking marijuana on focal attention and brain blood flow. Hum. Psychopharmacol. 22, 135-148 (2007).

61. Gorka, S. M. et al. Cannabinoid modulation of frontolimbic activation and connectivity during volitional regulation of negative affect. Neuropsychopharmacology 41, 1888-1896 (2016).

62. Gorka, S. M., Fitzgerald, D. A., de Wit, H. \& Phan, K. L. Cannabinoid modulation of amygdala subregion functional connectivity to social signals of threat. Int. J. Neuropsychopharmacol. https://doi.org/10.1093/ijnp/pyu104. (2014).

63. Phan, K. L. et al. Cannabinoid modulation of amygdala reactivity to social signals of threat in humans. J. Neurosci. 28, 2313-2319 (2008).

64. Rabinak, C. A., Sripada, C. S., Angstadt, M., de Wit, H. \& Phan, K. L. Cannabinoid modulation of subgenual anterior cingulate cortex activation during experience of negative affect. J. Neural Transm. 119, 701-707 (2012).

65. Rabinak, C. A., Peters, C., Marusak, H. A., Ghosh, S. \& Phan, K. L. Effects of acute $\Delta 9$-tetrahydrocannabinol on next-day extinction recall is mediated by postextinction resting-state brain dynamics. Neuropharmacology 143, 289-298 (2018).

66. Barkus, E. et al. Does intravenous $\Delta 9$-tetrahydrocannabinol increase dopamine release? A SPET study. J. Psychopharmacol. 25, 1462-1468 (2011).

67. Bhattacharyya, S. et al. Modulation of mediotemporal and ventrostriatal function in humans by $\Delta 9$-tetrahydrocannabinol: a neural basis for the effects of Cannabis sativa on learning and psychosis. Arch. Gen. Psychiatry 66, 442-451 (2009).

68. Bhattacharyya, S. et al. Opposite effects of delta-9-tetrahydrocannabinol and cannabidiol on human brain function and psychopathology. Neuropsychopharmacology 35, 764-774 (2010).

69. Bhattacharyya, S. et al. Induction of psychosis by $\Delta 9$-tetrahydrocannabinol reflects modulation of prefrontal and striatal function during attentional salience processing. Arch. Gen. Psychiatry 69, 27-36 (2012).

70. Borgwardt, S. J. et al. Neural basis of $\Delta$-9-tetrahydrocannabinol and cannabidiol: effects during response inhibition. Biol. Psychiatry 64, 966-973 (2008).

71. Fusar-Poli, P. et al. Distinct effects of $\Delta 9$-tetrahydrocannabinol and cannabidiol on neural activation during emotional processing. Arch. Gen. Psychiatry 66, 95-105 (2009).

72. Rzepa, E., Tudge, L. \& McCabe, C. The CB1 neutral antagonist tetrahydrocannabivarin reduces default mode network and increases executive control network resting state functional connectivity in healthy volunteers. Int. J. Neuropsychopharmacol. https://academic.oup.com/ijnp/article-abstract/ 19/2/pyv092/2910095. (2016).

73. Tudge, L., Williams, C., Cowen, P. J. \& McCabe, C. Neural effects of cannabinoid $C B 1$ neutral antagonist tetrahydrocannabivarin on food reward and aversion in healthy volunteers. Int. J. Neuropsychopharmacol. https://doi.org/ 10.1093/ijnp/pyu094. (2014).

74. Bhattacharyya, S. et al. Effect of Cannabidiol on medial temporal, midbrain and striatal dysfunction in people at clinical high risk of psychosis: a randomized clinical trial. JAMA Psychiatry 75, 1107-1117 (2018).

75. Seiferth, N. Y. et al. Increased neural response related to neutral faces in individuals at risk for psychosis. Neuroimage 40, 289-297 (2008).

76. Fryer, S. L. et al. Should I stay or should I go? FMRI Study of response inhibition in early illness schizophrenia and risk for psychosis. Schizophr. Bull. 45, 158-168 (2019).

77. Alonso-Solís, A. et al. Altered default network resting state functional connectivity in patients with a first episode of psychosis. Schizophr. Res. 139, 13-18 (2012).

78. de la Fuente-Sandoval, C., Favila, R., Gómez-Martin, D., Pellicer, F. \& GraffGuerrero, A. Functional magnetic resonance imaging response to experimental pain in drug-free patients with schizophrenia. Psychiatry Res. 183, 99-104 (2010). 
79. Ginovart, $N$. et al. Chronic $\Delta$ 9-tetrahydrocannabinol exposure induces a sensitization of dopamine $D_{2} / 3$ receptors in the mesoaccumbens and nigrostriatal systems. Neuropsychopharmacology 37, 2355-2367 (2012).

80. Ottani, A. \& Giuliani, D. HU 210: a potent tool for investigations of the cannabinoid system. CNS Drug Rev. 7, 131-145 (2006).

81. Nguyen, V. H. et al. Increased brain metabolism after acute administration of the synthetic cannabinoid HU210: a small animal PET imaging study with 18F-FDG. Brain Res. Bull. 87, 172-179 (2012).

82. Higuera-Matas, A. et al. Augmented acquisition of cocaine self-administration and altered brain glucose metabolism in adult female but not male rats exposed to a cannabinoid agonist during adolescence. Neuropsychopharmacology 33, 806-813 (2008).

83. Dalton, V. S. \& Zavitsanou, K. Differential treatment regimen-related effects of cannabinoids on D1 and D2 receptors in adolescent and adult rat brain. J. Chem. Neuroanat. 40, 272-280 (2010).

84. Howlett, A. C. et al. The cannabinoid receptor: biochemical, anatomical and behavioral characterization. Trends Neurosci. 13, 420-423 (1990).

85. John, W. S. et al. Chronic $\triangle 9-T H C$ in rhesus monkeys: effects on cognitive performance and dopamine D2/D3 receptor availability. J. Pharm. Exp. Ther. 364, 300-310 (2018).

86. Winton-Brown, T. et al. Modulation of auditory and visual processing by delta-9-tetrahydrocannabinol and cannabidiol: an FMRI study. Neuropsychopharmacology 36, 1340-1348 (2011).

87. Valdeolivas, S., Sagredo, O., Delgado, M., Pozo, M. A. \& Fernández-Ruiz, J. Effects of a sativex-like combination of phytocannabinoids on disease progression in R6/2 mice, an experimental model of Huntington's disease. Int. J. Mol. Sci. https://doi.org/10.3390/ijms18040684. (2017).

88. Stokes, P. R. A., Mehta, M. A., Curran, H. V., Breen, G. \& Grasby, P. M. Can recreational doses of $\mathrm{THC}$ produce significant dopamine release in the human striatum? Neuroimage 48, 186-190 (2009).

89. Ben-Jonathan, N. \& Hnasko, R. Dopamine as a prolactin (PRL) inhibitor. Endocr. Rev. 22, 724-763 (2001).

90. Howes, O. et al. Progressive increase in striatal dopamine synthesis capacity as patients develop psychosis: a PET study. Mol. Psychiatry 16 85-886 (2011). 8

91. Abi-Dargham, A. et al. Increased baseline occupancy of D2 receptors by dopamine in schizophrenia. Proc. Natl Acad. Sci. USA 97, 8104-8109 (2000).

92. Abi-Dargham, A. et al. Increased striatal dopamine transmission in schizophrenia: confirmation in a second cohort. Am. J. Psychiatry 155, 761-767 (1998)

93. Wang, $X$. et al. Disrupted resting-state functional connectivity in minimally treated chronic schizophrenia. Schizophr. Res. 156, 150-156 (2014).

94. Schutters, S. I. J. et al. The association between social phobia, social anxiety cognitions and paranoid symptoms. Acta Psychiatr. Scand. 125, 213-227 (2012).

95. Solowij, N. et al. Cognitive functioning of long-term heavy cannabis users seeking treatment. JAMA 287, 1123-1131 (2002).
96. de la Fuente-Sandoval, $C$. et al. Striatal glutamate and the conversion to psychosis: a prospective 1H-MRS imaging study. Int. J. Neuropsychopharmacol. 16, 471-475 (2013)

97. Taconic Biosciences, Inc. Sprague Dawley Rat. https:/www.taconic.com/ pdfs/sprague-dawley-rat.pdf.

98. Cremers, H. R., Wager, T. D. \& Yarkoni, T. The relation between statistical power and inference in fMRI. PLoS One 12, e0184923 (2017).

99. Turner B. O., Paul E. J., Miller M. B., Barbey A. K. Small sample sizes reduce the replicability of task-based fMRI studies. Communications Biology. 2018; 1. https://doi.org/10.1038/s42003-018-0073-z.

100. Haensel, J. X., Spain, A. \& Martin, C. A systematic review of physiological methods in rodent pharmacological MRI studies. Psychopharmacology 232 489-499 (2015).

101. Beuf, O., Jaillon, F., Saint-Jalmes, H. \& Small-animal, M. R. I. signal-to-noise ratio comparison at 7 and $1.5 \mathrm{~T}$ with multiple-animal acquisition strategies. MAGMA 19, 202-208 (2006)

102. Ohlsson, A. et al. Plasma delta-9 tetrahydrocannabinol concentrations and clinical effects after oral and intravenous administration and smoking. Clin. Pharm. Ther. 28, 409-416 (1980).

103. Button, K. S. et al. Power failure: why small sample size undermines the reliability of neuroscience. Nat. Rev. Neurosci. 14, 365-376 (2013).

104. Hasan A., et al. Cannabis use and psychosis: a review of reviews. Eur Arch Psychiatry Clin Neurosci 2019. https://doi.org/10.1007/s00406-019-01068-z

105. Mwansisya, T. E. et al. Task and resting-state fMRI studies in first-episode schizophrenia: A systematic review. Schizophr. Res 189, 9-18 (2017).

106. Whitfield-Gabrieli, S. \& Ford, J. M. Default mode network activity and connectivity in psychopathology. Annu Rev. Clin. Psychol. 8, 49-76 (2012).

107. Sepede, G. et al. Sustained attention in psychosis: Neuroimaging findings. World J. Radio. 6, 261-273 (2014).

108. Craft, R. M. Sex differences in behavioral effects of cannabinoids. Life Sci. 77 2471-2478 (2005).

109. Craft, R. M., Marusich, J. A. \& Wiley, J. L. Sex differences in cannabinoid pharmacology: a reflection of differences in the endocannabinoid system? Life Sci. 92, 476-481 (2013).

110. Donoghue, K. et al. Cannabis use, gender and age of onset of schizophrenia: data from the AESOP study. Psychiatry Res 215, 528-532 (2014).

111. Rubino, T. \& Parolaro, D. Sexually dimorphic effects of cannabinoid compounds on emotion and cognition. Front Behav. Neurosci. 5, 64 (2011).

112. Rubino, T. et al. Chronic delta 9-tetrahydrocannabinol during adolescence provokes sex-dependent changes in the emotional profile in adult rats: behavioral and biochemical correlates. Neuropsychopharmacology 33 2760-2771 (2008)

113. Viveros, M. P. et al. The endocannabinoid system in critical neurodevelopmental periods: sex differences and neuropsychiatric implications. J. Psychopharmacol. 26, 164-176 (2012).

114. Head, M. L., Holman, L., Lanfear, R., Kahn, A. T. \& Jennions, M. D. The extent and consequences of $\mathrm{p}$-hacking in science. PLOS Biol. 13, e1002106 (2015). 